\title{
Zweifach wirksam bei extrem trockener Haut und Neurodermitis
}

\begin{abstract}
- Atopische Haut spannt, neigt zu Juckreiz und macht den Betroffenen das Leben schwer. Helfen kann eine konsequente Behandlung, die den Wiederaufbau der pathologisch gestörten Hautschutzbarriere fördert sowie Juckreiz und Irritationen lindert.

Mit Lipikar Körperbalsam AP von La RochePosay steht eine neue, intensiv rückfettende und hydratisierende Formel zur Verfügung, die sich die positiven Eigenschaften von hochkonzentriertem Niacinamid (4\%) zu Nutze macht. Ein wissenschaftliches
\end{abstract}

Gutachten von Prof. Dr. Johannes Wohlrab, Leitender Oberarzt der Universitätsklinik und Poliklinik für Dermatologie und Venerologie in Halle an der Saale, bestätigt die verschiedenen Wirkansätze und gute Verträglichkeit des Vitamin-B3 Niacinamid.

Wie sehr diese positive Wirkung des Körperbalsams das Wohlbefinden der Patienten steigern kann, zeigt eine kanadische Multicenterstudie mit 73 Kindern im Alter von 3 bis 12 Jahren, die an leichter bis mittlerer atopischer Dermatitis litten.
In der klinischen Studie, durchgeführt von Prof. Dr. Robert Bissonnette, Universität Montreal, Kanada, erwies sich Lipikar Körperbalsam A therapiebegleitend als sehr gut hautverträglich und deutlich Juckreiz lindernd (Rückgang des SCORAD-Index um bis zu 57\%). Der Schlafmangel der jungen Atopiker ging um $72 \%$ zurück, der Zustand der Haut wurde als deutlich verbessert empfunden. Auch die kosmetische Qualität des Körperbalsams wurde sehr positiv beurteilt: $76 \%$ der Kinder (bzw. der beteiligten Erwachsenen) bestätigten die praktische Anwendung, und $94 \%$ lobten die leichte Verteilbarkeit des Balsams.

red

Nach Informationen von La Roche-Posay

\section{Atopisches Ekzem: Mit proaktiver Therapie Rezidive vermeiden}

- Häufig auftretende Rezidive erschweren das Langzeitmanagement des atopischen Ekzems (AE). Vor allem in der Akuttherapie haben sich topische Kortikosteroide bislang als wirksam und sicher erwiesen. Jüngere Studien haben gezeigt, dass eine proaktive Therapie mit Methylprednisolonaceponat (Advantan $\left.{ }^{\circledR}\right)$ dazu beiträgt, die Krankheitsaktivität beim atopischen Ekzem langfristig zu kontrollieren. Rezidive können so vermieden und die Lebensqualität der Patienten dauerhaft verbessert werden.

In einer randomisierten, doppelblinden, kontrollierten Multicenter-Studie konnte gezeigt werden, dass eine zweimal wöchentlich durchgeführte Erhaltungstherapie mit Methylprednisolonaceponat (MPA) effektiv dazu beiträgt, die Rezidivhäufigkeit zu verringern. In die Untersuchung wurden 249 AE-Patienten ab 12 Jahren eingeschlossen, die unter einem schweren oder sehr schweren akuten AE-Schub litten (Investigator Global Assessment-Score >4). Nach erfolgreicher Akutbehandlung wurden 221 Patienten in die Erhaltungsphase übernommen. Sie erhielten über 16 Wochen entweder zweimal wöchentlich $0,1 \%$ ige MPA-Creme plus Emolliens ( $\mathrm{n}=$ 112 ) oder nur Emolliens ( $n=109)$. Primärer Endpunkt der Studie war die Zeit bis zu einem Rezidiv. Es zeigte sich, dass der proaktive Therapieansatz mit MPA zu einer 3,5-mal geringeren Rezidivrate führte. Die Wahrscheinlichkeit, auch nach 16 Wochen noch rezidivfrei zu bleiben, lag in der proaktiven MPA-Gruppe bei $87,1 \%$ und in der Emolliens-Gruppe bei 65,8\%.

Advantan ${ }^{\circledR}$ ist ein topisches Glukokortikoid der 4. Generation. MPA wird in erster Linie zur Behandlung des endogenen Ekzems
- Seit Anfang dieses Jahres ist mit Juvéderm ${ }^{\circledR}$ ULTRA SMILE ein neuer Hyaluronsäurefiller speziell zur Konturierung und Modellierung der Lippen erhältlich. Anstatt aus einer granulatartigen Textur, wie andere Filler, besteht das Produkt aus einer weiterentwickelten 3D-Hyaluronsäure-Matrix mit hoher Bindekraft für Wasser. Durch die Verwendung der natürlichen Substanz wird potentiellen Allergierisiken vorgebeugt. Dem Filler wurde zudem ein lokal wirkendes, leichtes Betäubungsmittel zugegeben. Das Produkt wird in kleinen Dosierungen in die Lippen injiziert und gibt diesen Form und Volumen sowie ein ebenmäßiges Aussehen. Das geschmeidigen Gel fühlt sich auch beim Küssen natürlich an. In aktuellen eingesetzt. Aufgrund seiner hohen Lipophilie kann der Wirkstoff schnell durch die Haut penetrieren. Durch enzymatische Hydrolyse wird MPA rasch in Methylprednisolon-17-propionat (MPP) umgewandelt, das eine 2,4-fach höhere Bindungsaffinität zu den Kortikoidrezeptoren besitzt. Advantan ${ }^{\circledR}$ ist in 5 verschiedenen Galeniken erhältlich - als Creme, Salbe, Lösung, Fettsalbe oder Milch.

red

Nach Informationen von Intendis

\section{Neuer Filler für die Lippen}

Studien waren 97\% der mit dem Filler behandelten Patienten der Meinung, er gebe ihnen natürlich aussehende Lippen; 91\% äußersten sich zufrieden mit dem Behandlungsergebnis. Die Juvéderm ${ }^{\circledR}$ Familie gründete sich auf eine Reihe, die für die Behandlung von moderaten bis tiefen Gesichtsfalten und zum Aufbau von neuem Volumen eingesetzt wird. Mit drei verschiedenen Darreichungsformen steht für jede Faltentiefe und Form des Volumenverlusts das passende Produkt zur individuellen Behandlung zur Verfügung. Alle Produkte zeichnen sich durch das integrierte Betäubungsmittel Lidocain aus. red

Nach Informationen von Allergan 